02

\title{
Влияние направления внешнего магнитного поля на зонную структуру магнитофотонных кристаллов
}

\author{
(C) А.А. Геворгян ${ }^{1}$, С.С. Голик ${ }^{2}$, Т.А. Геворгян ${ }^{2}$ \\ ${ }^{1}$ Ереванский государственный университет, \\ 0025 Ереван, Армения \\ 2 Дальневосточный федеральный университет, \\ 690091 Владивосток, Россия \\ e-mail: agevorgyan@ysu.am,golik.ss@dvfu.ru,gevorgian.ta@students.dvfu.ru
}

Поступила в редакцию 24.10.2017 г.

\begin{abstract}
Исследованы особенности зонной структуры магнитофотонных кристаллов. Задача распространения света через конечный слой магнитофотонного кристалла решена модифицированным методом сложения слоев Амбарцумяна. Детально исследовано влияние направления внешнего магнитного поля на зонную структуру и показано, что оно приводит к изменениям числа запрещенных зон, частотной ширины этих зон, а также к изменению поляризационных особенностей этих зон. Показано, что такие системы имеют четыре собственные поляризации.
\end{abstract}

DOI: $10.21883 /$ OS.2018.03.45651.244-17

\section{Введение}

Интерес исследователей к магнитофотонным кристаллам (МФК) постоянно увеличивается. Это в первую очередь обусловлено тем обстоятельством, что МФК являются фотонными кристаллами (ФК) с легко управляемыми параметрами и находят все возрастающее применение в фотонике, оптике и в других направлениях науки и техники. В спектре пропускания МФК существуют фотонные запрещенные зоны (ФЗ3), частотные ширины и частотные местоположения, а также амплитудные и поляризационные свойства которых можно изменить, в частности, внешним магнитным полем (изменяя его величину или направление). Применение внешнего магнитного поля к пластинке из однородной магнитоактивной среды обычно обеспечивает лишь незначительное модулирование интенсивности света или ее поляризации, поэтому искали различные стратегии для усиления магнитооптических эффектов.

В последние годы для усиления магнитооптических эффектов предлагался метод управляемого наноструктурирования, в котором оптические свойства структуры в основном определяются геометрическими резонансами, а не электронными.

Другой класс легко управляемых МФК - магнитная жидкость, являющаяся своего рода стабильным коллоидным раствором, состоящим из наноразмерных магнитных частиц, покрытых поверхностно-активным веществом. Она обладает как усиленными магнитооптическими свойствами МФК, так и текучестью жидкостей, которые показывают множество магнитооптических характеристик, включая вращение Фарадея, настраиваемый показатель преломления и магнитное двулучепреломление [1-3]. В структурах с плазменным резонансом и магнитооптической активностью вблизи плазменного возбуждения также наблюдаются высокие значения маг- нитооптической активности и глубины модуляции [4-8]. Отметим, что существуют также МФК естественного происхождения, примерами которых являются монокристаллические пленки ферритов-гранатов с регулярной доменной структурой. В указанных структурах мы можем иметь дело с большими глубинами модуляции и большими значениями параметра магнитооптической активности. К тому же такие МФК могут иметь очень малые размеры, они способны обеспечить уникальные оптические и магнитооптические свойства запрещенных зон, невзаимности и дефектных мод [9-27].

В работах $[28,29]$ начато систематическое исследование свойств МФК с большой магнитооптической активностью и глубиной модуляции. В частности, показано, что при наклонном падении света возникает Ф3З нового типа, а также наблюдается невзаимность нового типа. В настоящей работе мы будем исследовать влияние направления внешнего статического магнитного поля на зонную структуру и собственные поляризации $(\mathrm{CП})$ указанных сред.

\section{Результаты}

Рассмотрим наклонное падение света на слой МФК толщиной $d$. Будем предполагать следующие законы изменения параметров среды:

$$
\left(\begin{array}{c}
\varepsilon(z) \\
\mu(z) \\
g(z)
\end{array}\right)=\left(\begin{array}{c}
\varepsilon \\
\mu \\
g
\end{array}\right)\left[1+\left(\begin{array}{c}
\Delta \varepsilon \\
\Delta \mu \\
\Delta g
\end{array}\right) \sin K z\right],
$$

где $\varepsilon, \mu$ - диэлектрическая и магнитная проницаемости, $g$ - параметр магнитооптической активности, $\Delta \varepsilon, \Delta \mu$, $\Delta g$ - глубины модуляции соответственно параметров $\varepsilon$, $\mu, g$ (они здесь могут быть значительными, но меньшими 
единицы), $K=\frac{2 \pi}{\Lambda}-$ период пространственной модуляции, $z$ - ось среды. Задачу прохождения света через такой слой МФК будем решать модифицированным методом сложения слоев Амбарцумяна [28,29].

Как известно, в случае однородного МФК с постоянным параметром магнитооптической активности наличие внешнего магнитного поля приводит к сдвигу пиков Ф33 для лево- и правоциркулярно поляризованного света в противоположные направления вдоль оси частоты. Далее в [28,29] показано, что при наклонном падении при больших значениях магнитооптической активности появляется новая Ф3З в каждом порядке дифракционного отражения, которая при условии $\theta=0(\theta-$ угол между направлением внешнего магнитного поля и осью среды) является не селективной по отношению к поляризации падающего света. Ниже будем исследовать влияние изменения направления внешнего магнитного поля (изменения угла $\theta$ ) на зонную структуру МФК при наклонном падении света в ситуации, когда формируются три области Ф3З в каждом порядке дифракционного отражения: две области - селективные по отношению к поляризации падающей волны и имеющие ортогональные циркулярные поляризации и одна - не селективная, находящая между Ф33, селективными по отношению к поляризации падающей волны.

На рис. 1 представлены спектры отражения при наклонном падении света (угол падения $\alpha=60^{\circ}$ ) и при большом значении параметра магнитооптической активности $g(g=0.8)$ при различных значениях угла $\theta$. Падающий на слой МФК свет имеет $s$ - (кривая 1 ) и $p$ - (кривая 2) линейные поляризации и левую (кривая 3) и правую (кривая 4) циркулярные поляризации (рис. $1, a, c, e, g, i, k, m$ ), а также первую и вторую СП (рис. $1, b, d, f, h, j, l, n)$. Собственные поляризации данной системы - это соответствующие поляризации падающей волны, не изменяющиеся при ее прохождении через данную систему. Как было сказано выше, в случае наклонного падения света при больших значениях параметра $g$ возникает новая область дифракционного отражения, которая при $\theta=0$ является поляризационно нечувствительной (на рис. $1, a, b$ она обозначена стрелкой). Как только $\theta$ начинает отличаться от нуля, эта область становится поляризационно чувствительной она расщепляется на две поляризационно чувствительные области с квазикруговыми поляризациями. С дальнейшим увеличением угла $\theta$ эти области смещаются в противоположные стороны (на рис. $1, c, d, e, f$ эти области обозначены стрелками). Далее происходит сложное преобразование зонной структуры - эти области сливаются с коротковолновой и длинноволновой областями с квазилевой и квазиправой круговыми поляризациями. Одновременно происходит значительное изменение СП. Наконец, при $\theta=90^{\circ}$ формируются две области в каждом порядке дифракционного отражения - одна, длинноволновая - не селективная, и другая, коротковолновая - с квазиправой круговой поляризацией.
Для более полной информации на рис. 2 представлена эволюция спектров отражения при изменении угла падения $\alpha$ при разных значениях угла $\theta$ (угла между внешним магнитным полем и осью среды (осью $z$ )). Падающий свет имеет $s$ - (рис. $2, a, c, e, g, i)$ и $p$ - (рис. $2, b, d, f, h, j)$ линейные поляризации. Рис. $2, a, b$ соответствует случаю $\theta=0$, рис. $2, c, d-$ случаю $\theta=30^{\circ}$, рис. $2, e, f-$ случаю $\theta=45^{\circ}$, рис. $2, g, h-$ случаю $\theta=60^{\circ}$ и, наконец, рис. $2, i, j$ - для $\theta=90^{\circ}$.

На рис. 3 представлена эволюция спектров отражения в тех же случаях, что и на рис. 2 , но при условии, что падающий свет имеет левую (рис. $3, a, c, e, g, i$ ) и правую (рис. $3, b, d, f, h, j$ ) круговые поляризации.

Далее исследовали особенности эволюции спектров отражения при изменении угла $\theta$ для различных углов падения света $\alpha$. На рис. 4 представлена эволюция спектров отражения при изменении угла $\theta$ при разных значениях угла падения $\alpha$. Падающий на слой МФК свет имеет $s$ - (рис. $4, a, c, e, g, i)$ и $p$ - (рис. $4, b, d, f, h, j)$ линейные поляризации. Рисунок $4, a, b$ соответствуют случаю $\alpha=0$, рис. $4, c, d-$ случаю $\alpha=30^{\circ}$, рис. $4, e, f-$ случаю $\alpha=45^{\circ}$, рис. $4, g, h-$ случаю $\alpha=60^{\circ}$ и, наконец, рис. $4, i, j-$ случаю $\alpha=85^{\circ}$.

На рис. 5 представлена эволюция спектров отражения в тех же случаях, что и на рис. 4 , но когда падающий свет имеет левую (рис. $5, a, c, e, g, i$ ) и правую (рис. $5, b, d, f, h, j$ ) круговые поляризации. Как видно из рис. 4,5 , изменение направления внешнего магнитного поля при неизменной величине этого поля приводит к множеству эффектов: к изменению характера ФЗ3 - она превращается из поляризационно нечувствительной в чувствительную к поляризации и, наоборот, к изменению частотных положений ФЗ3, к изменению ширин полос Ф33, к увеличению числа ФЗЗ в каждом порядке дифракционного отражения, к уменьшению числа ФЗ3 - при определенных значениях угла $\theta$ Ф3З вообще исчезают в каждом порядке дифракционного отражения и при некоторой поляризации падающего света. Представленные рисунки демонстрируют уникальную возможность управления светом в широких пределах путем настройки одного только направления внешнего магнитного поля.

Далее, будем исследовать влияние изменения направления внешнего магнитного поля на СП. Как известно, изотропные, анизотропные, гиротропные, взаимные среды и ФК в виде плоскопараллельных слоев имеют две СП. В определенных случаях эти СП могут быть вырожденными, т.е. могут совпадать. Например, при нормальном падении света на плоскопараллельный слой из изотропной среды любая поляризация падающего света собственная, т.е. не изменяется при его прохождении через систему. Собственные поляризации вырождены также в многослойной системе из правои левовращающих идентичных слоев холестерического жидкого кристалла с четным числом подслоев. Ситуация иная в случае невзаимных сред. Для таких сред СП при падении света на слой справа могут отличатся от СП при его падении с обратной стороны. Однако по нашим 

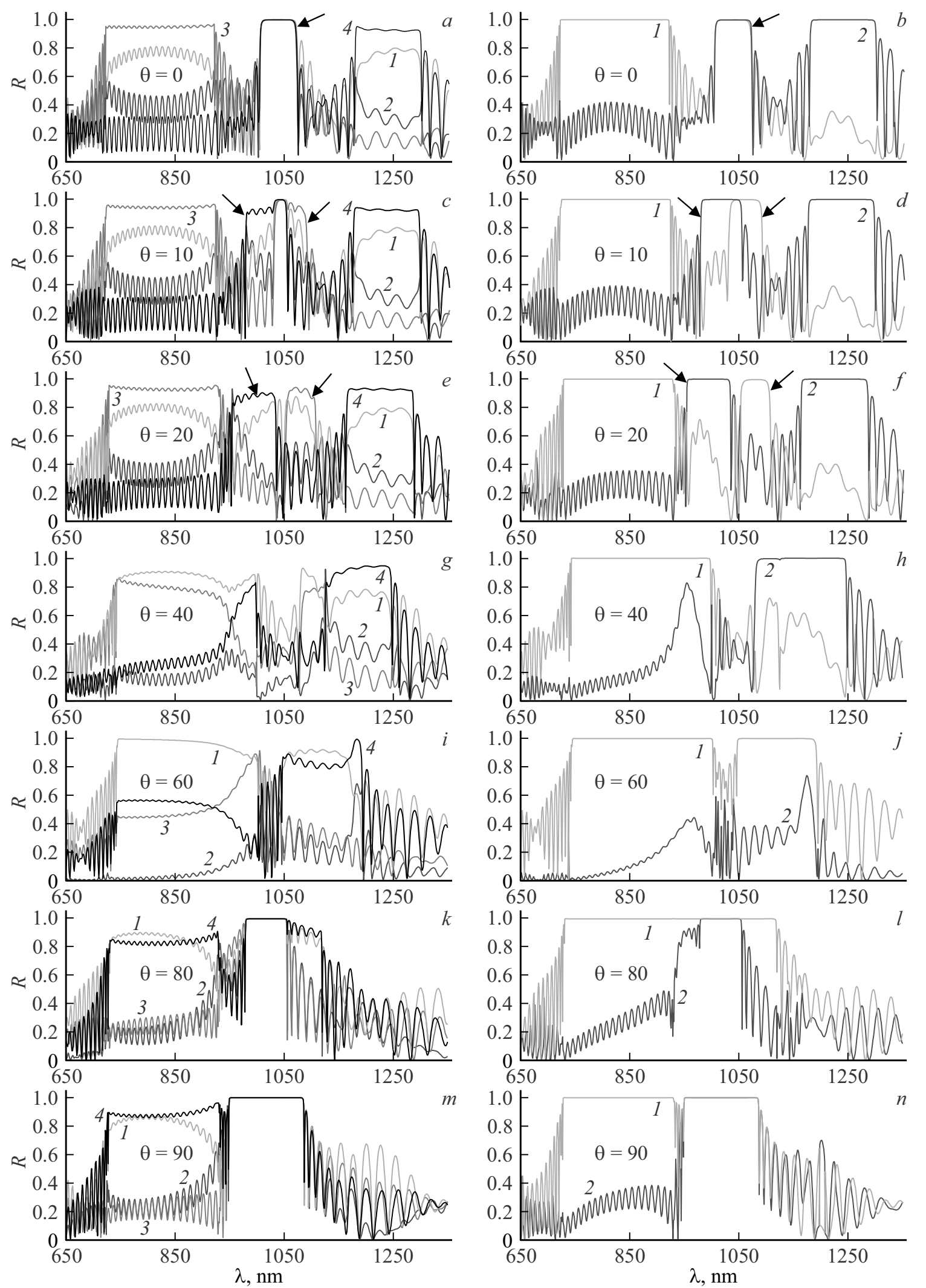

Рис. 1. Спектры отражения при наклонном падении света $\left(\alpha=60^{\circ}\right)$ при больших значениях постоянной магнитооптической активности $g(g=0.8)$ и различных углах $\theta$ (указаны на рисунке). Падающий на слой МФК свет имеет $s$ - $(1)$ и $p$ - $(2)$ линейные поляризации и левую (3) и правую (4) циркулярные поляризации $(a, c, e, g, i, k, m)$, а также первую и вторую СП $(b, d, f, h, j, l, n)$. Другие параметры следующие: диэлектрическая проницаемость $\varepsilon=2.5$, ее глубина модуляции $\Delta \varepsilon=0.7$, магнитная проницаемость $\mu(z)=1$, пространственный период модуляции $\Lambda=400 \mathrm{~nm}$, толщина слоя $d=50 \Lambda$, показатель преломления сред окружающих с двух сторон слой МФК $n_{0}=1$. 

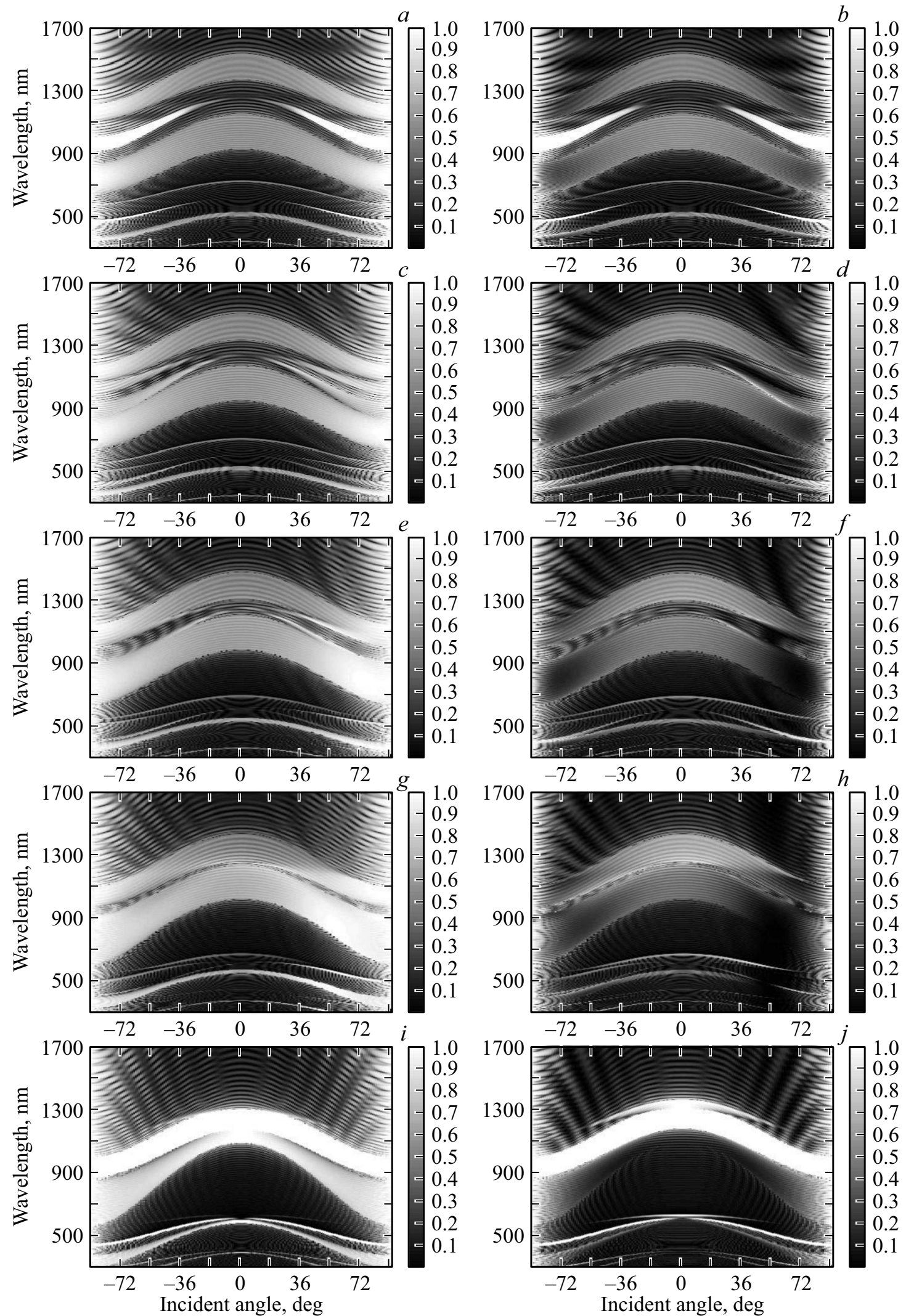

Рис. 2. Эволюция спектров отражения при изменении угла падения $\alpha$ при $\theta=0(a, b), 30^{\circ}(c, d), 45^{\circ}(e, f), 60^{\circ}(g, h), 90^{\circ}(i, j)$. Падающий свет имеет $s$ - $(a, c, e, g, i)$ и $p$ - $(b, d, f, h, j)$ линейные поляризации. Другие параметры такие же, что и на рис. 1. 

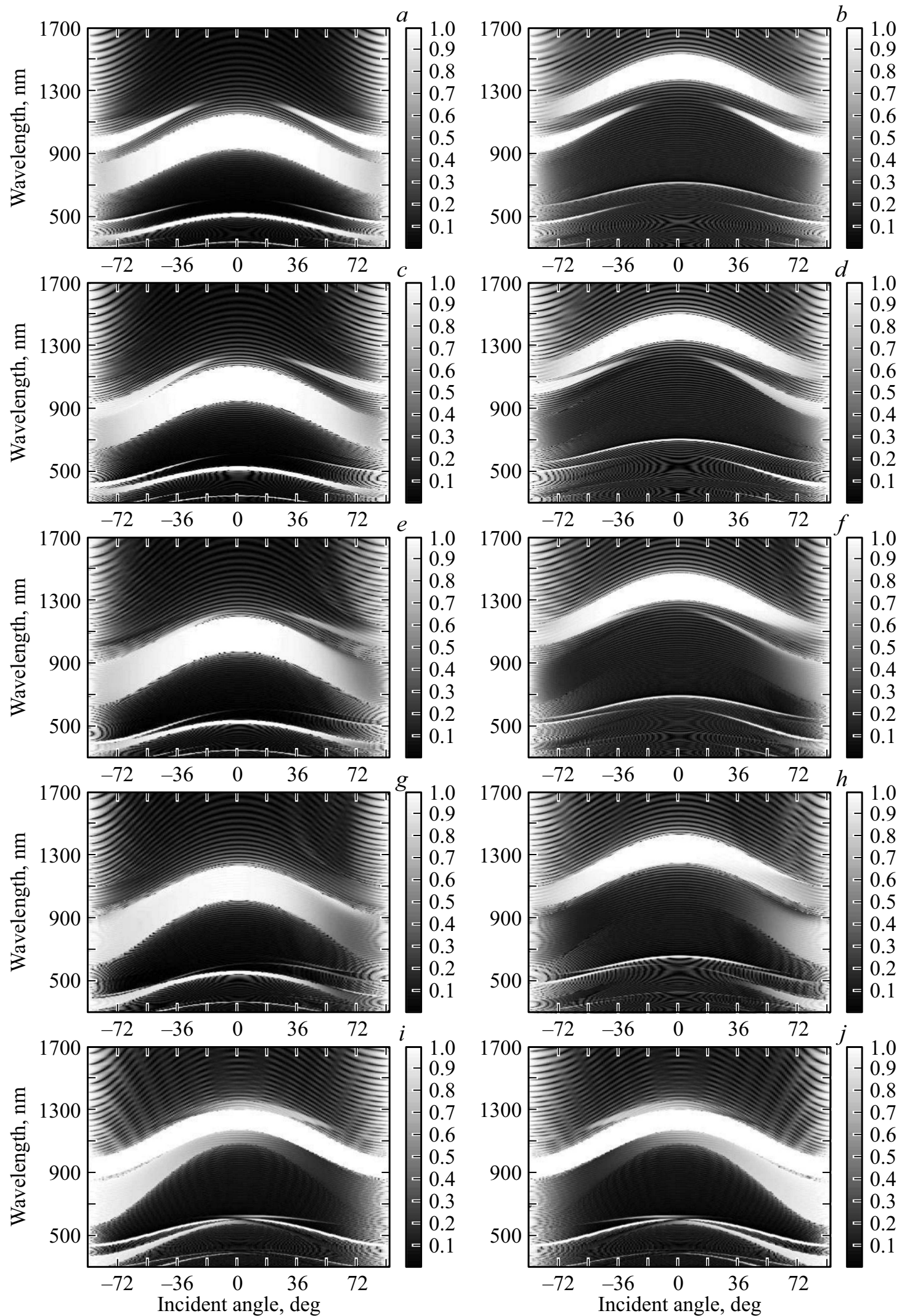

Рис. 3. Эволюция спектров отражения в тех же случаях, что и на рис. 2 , но падающий свет имеет правую $(a, c, e, g, i)$ и левую $(b, d, f, h, j)$ круговые поляризации. 

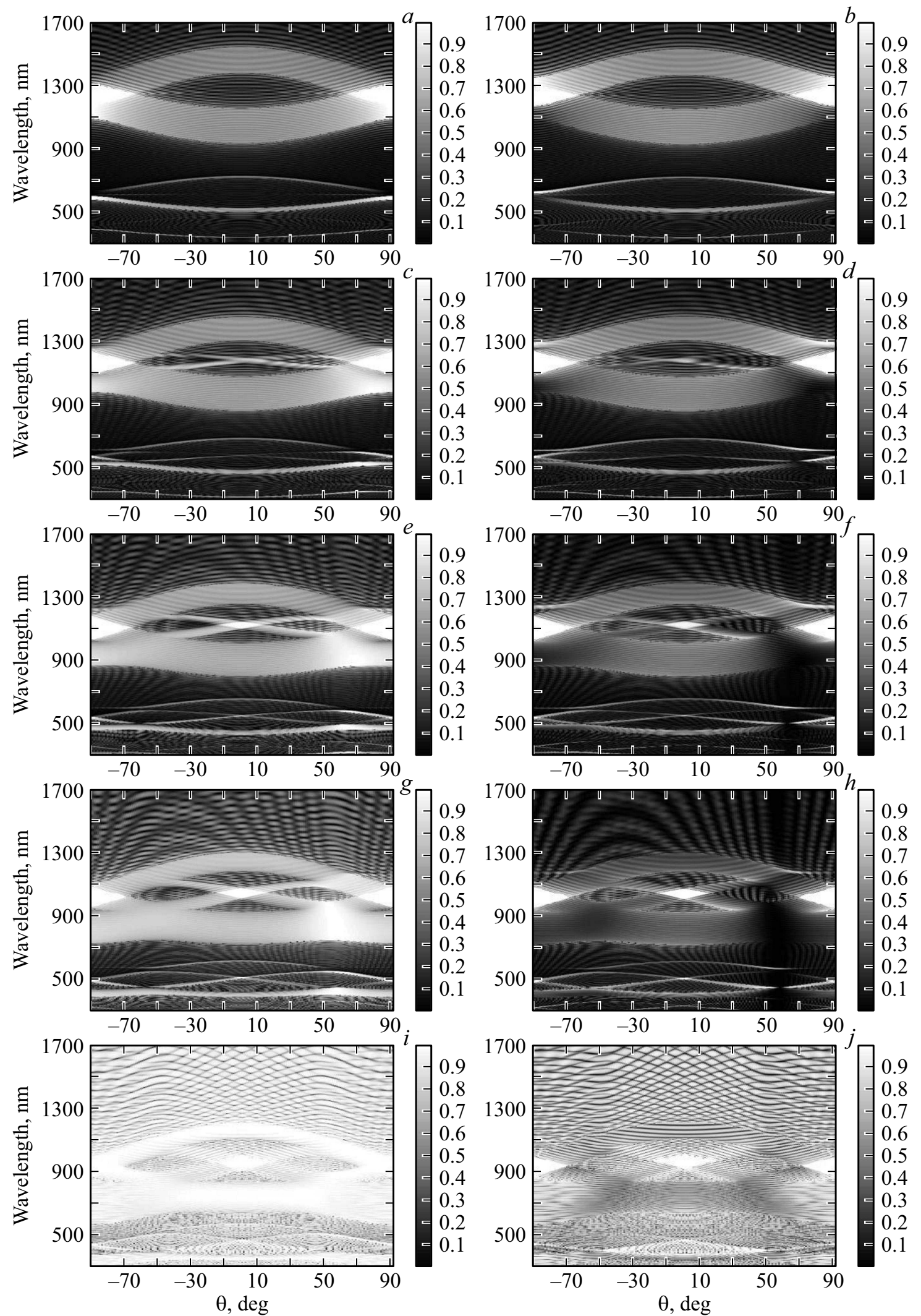

Рис. 4. Эволюция спектров отражения при изменении угла $\theta$ при различных значениях угла падения $\alpha$. Падающий свет имеет $s-(a, c, e, g, i)$ и $p$ - $(b, d, f, h, j)$ линейные поляризации. $\alpha=0(a, b), 30^{\circ}(c, d), 45^{\circ}(e, f), 60^{\circ}(g, h), 85^{\circ}(i, j)$. Другие параметры такие же, как на рис. 1. 

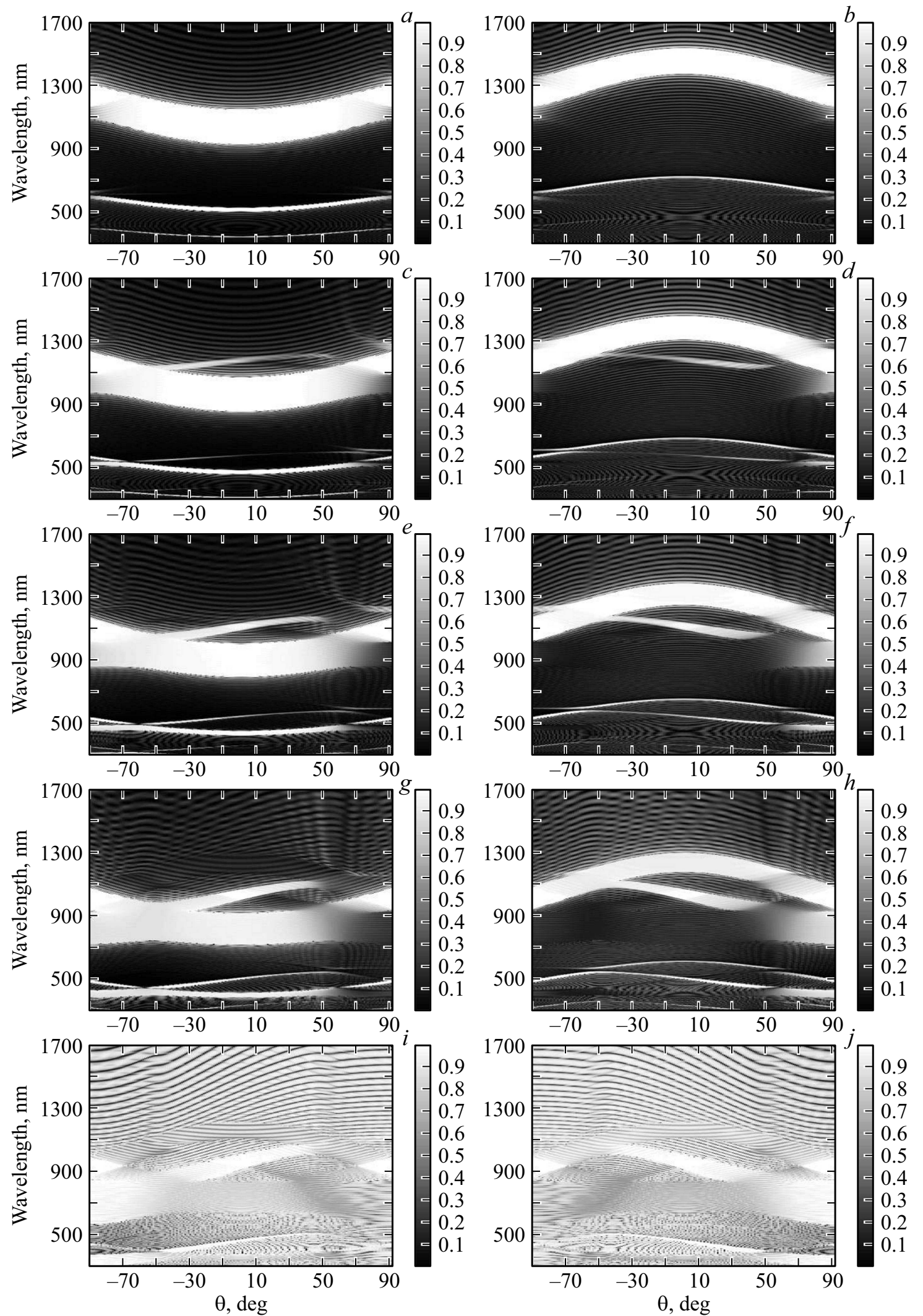

Рис. 5. Эволюция спектров отражения в тех же случаях, что и на рис. 4, но падающий свет имеет правую $(a, c, e, g, i)$ и левую $(b, d, f, h, j)$ круговые поляризации. 

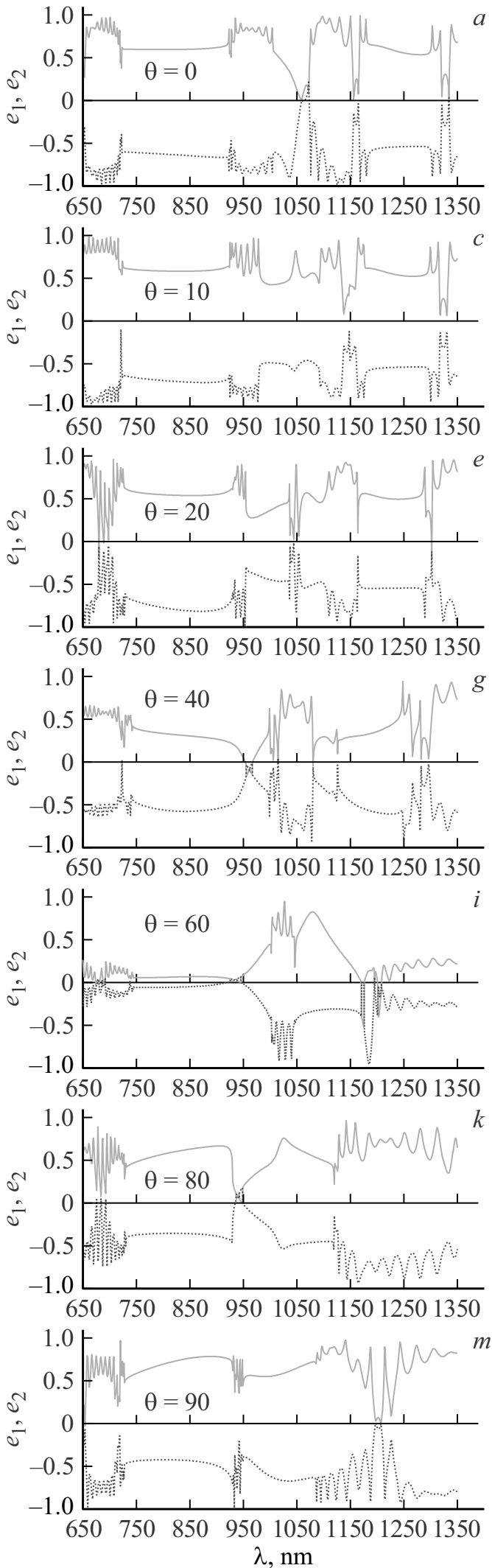
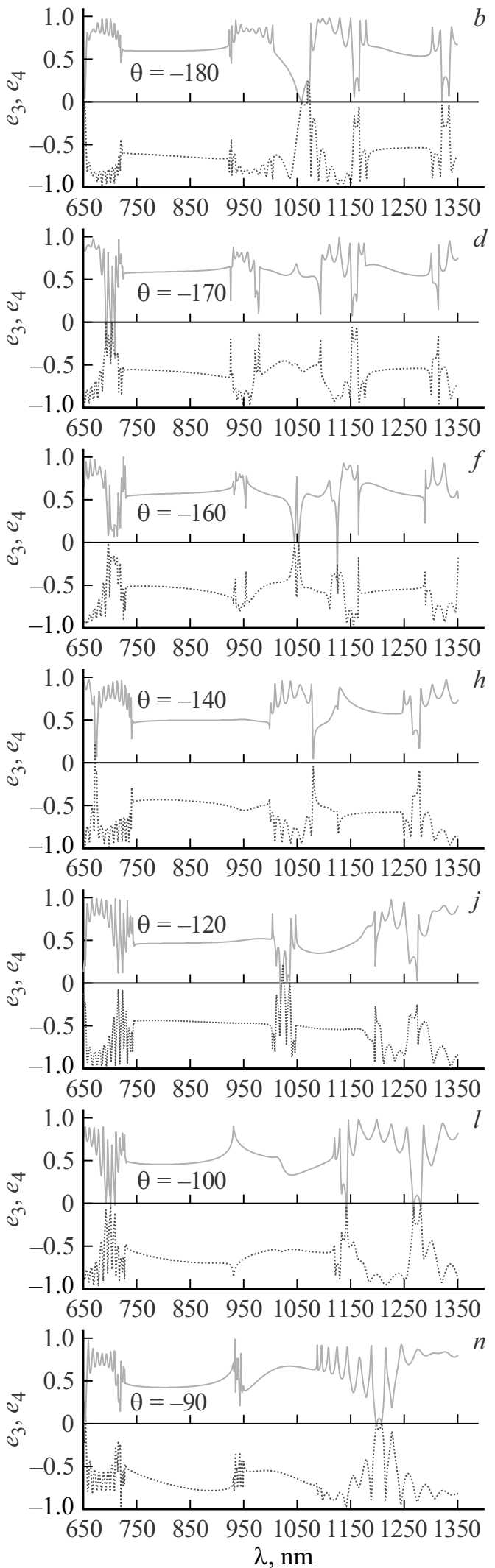

Рис. 6. Спектры эллиптичностей СП при наклонном падении света (угол падения $\alpha=60^{\circ}$ ) и при большом значении параметра магнитооптической активности $g(g=0.8)$ при различных значениях угла $\theta$ (указанных на рисунках) в случаях падения света на систему соответственно с левой $(a, c, e, g, i, k, m)$ и с правой $(b, d, f, h, j, l, n)$ сторон. Параметры такие же, что и на рис. 1. 
данным, на это обстоятельство не обращают внимания, или это отличие было настолько незначительным, что не представляло большого интереса. Однако, как показывают наши исследования, для исследуемой системы это отличие может стать существенно большим.

На рис. 6 представлены спектры эллиптичностей СП при наклонном падении света (угол падения $\alpha=60^{\circ}$ ), при большом значении параметра магнитооптической активности $g(g=0.8)$ и при различных значениях угла $\theta$ в случаях падения света на систему соответственно с левой (рис. $6, a, c, e, g, i, k, m$ ) и с правой (рис. $6, b, d, f, h, j, l, n$ ) сторон. Как видно из этих рисунков, при $\theta=0$ и $90^{\circ}$ разность между СП при падении света с левой и с правой сторон незначительная. Однако при других значениях угла $\theta$ данная разность может быть очень большой. Таким образом, можем утверждать, что невзаимные системы имеют четыре СП в отличие от взаимных плоскопараллельных структур.

Кроме того, отметим, что существуют системы и с большим числом СП. Так, например, в работе [30] изучена метаповерхность, состоящая из наклонных ахиральных метаатомов без пространственного изменения элементарной ячейки, которая показывает заметную оптическую хиральность исключительно из асимметричных взаимодействий между метаатомами. В такой системе авторами измерены шесть СП мод.

\section{Заключение}

В заключение отметим, что нами исследовано влияние направления внешнего магнитного поля на оптические свойства МФК и показана возможность управления в широких пределах оптическими свойствами МФК изменением одного лишь направления магнитного поля. Показано, что изменение направления внешнего магнитного поля приводит к кардинальному изменению зонной структуры МФК. Установлено, что в отличие от изотропных, анизотропных, гиротропных, взаимных сред и ФК в виде плоскопараллельных слоев эти системы имеют не две, а четыре, в общем случае существенно отличающихся друг от друга СП.

Голик С.С. и Геворгян Т.А. благодарят за финансовую поддержку работы Российский научный фонд (соглашение 14-50-00034).

\section{Список литературы}

[1] Fan F., Chen S., Wang X.H., Wu P.F., Chang S.J. // Appl. Phys. Lett. 2013. V. 103. N 16. P. 161115.

[2] Chen S., Fan F., Wang X.-H., Chang S.-J. // Opt. Expr. 2014. V. 22. N 6. P. 6313.

[3] Brojabasi S., Muthukumaran T., Laskar J.M., Philip J. // Opt. Commun. 2015. V. 336. P. 278.

[4] Armelles G., Cebollada A., García-Martín A., González M.U. // Adv. Opt. Mater. 2013. V. 1. P. 10.
[5] Hermann C., Kosobukin V.A., Lampel G., Peretti J., Safarov V.I., Bertrand P. // Phys. Rev. B. 2001. V. 64. P. 235422.

[6] González-Díaz J.B., García-Martín A., García-Martín J.M., Cebollada A., Armelles G., Sepúlveda B., Alaverdyan Y., Käll M. // Small. 2008. V. 4. N 2. P. 202.

[7] Belotelov V.I., Akimov I.A., Pohl M., Kotov V.A., Kasture S., Vengurlekar A.S., Gopal A.V., Yakovlev D.R., Zvezdin A.K., Bayer M. // Nat. Nanotechnol. 2011. V. 6. P. 370.

[8] Chin J., Steinle T., Wehlus T., Dregely D., Weiss T., Belotelov V.L., Stritzker B., Giessen H. // Nat. Commun. 2013. V. 4. P. 1599.

[9] Belotelov V.I., Zvezdin A.K. // J. Opt. Soc. Am. B. 2005. V. 22. N 1. P. 286.

[10] Inoue M., Fujikawa R., Baryshev A., Khanikaev A., Lim P.B., Uchida H., Aktsipetrov O., Fedyanin A., Murzina T., Granovsky A. // J. Phys. D. 2006. V. 39. N 8. P. R151.

[11] Grishin A.M., Khartsev S.I. // J. Magn. Soc. Jpn. 2008. V. 32. P. 140.

[12] Gevorgyan A.H. // Mol. Cryst. Liq. Cryst. 2002. V. 382. P. 1.

[13] Figotin A., Vitebskiy I. // Phys. Rev. B. 2003. V. 67. P. 165210.

[14] Khanikaev A.B., Steel M.J. // Opt. Expr. 2009. V. 17. N 7. P. 5265.

[15] Геворгян А.А. // ЖТФ. 2002. Т. 72. № 8. С. 77. (Gevorgyan A.H. // Tech. Phys. 2002. V. 47. N 8. P. 1008.)

[16] Merzlikin A.M., Levy M., Vinogradov A.P., Wu Z., Jalali A.A. // Opt. Commun. 2010. V. 283. P. 4298.

[17] Jonsson F., Flytzanis C. // J. Opt. Soc. Am. B. 2005. V. 22. N 1. P. 293.

[18] Lyubchanskii I.L., Dadoenkova N.N., Lyubchanskii M.I., Shapovalov E.A., Rasing T. // J. Phys. D. 2003. V. 36. N 11. P. R277.

[19] Арутюнян Э.М., Геворгян А.А. // ЖТФ. 2009. Т. 79. № 1. C. 98. (Harutyunyan E.M., Gevorgyan A.H. // Tech. Phys. 2009. V. 54. N 1. P. 95.)

[20] Zhang H.F., Liu S.B., Kong X.K., Li B.X. // Eur. Phys. J. D. 2013. V. 67. P. 169.

[21] Kato H., Matsushita T., Takayama A., Egawa M., Nishimura K., Inoue M. // IEEE Trans. Magn. 2002. V. 38. N 5. P. 3246.

[22] Smigaj W., Romero-Vivas J., Gralak B., Magdenko L., Dagens B., Vanwolleghem M. // Opt. Lett. 2010. V. 35. N 4. P. 568.

[23] Wang Q., Ouyang Z., Liu Q. // J. Opt. Soc. Am. B. 2011. V. 28. N 4. P. 703.

[24] Елисеева С.В., Семенцов Д.И. // ФТТ. 2012. Т. 54. № 10. C. 1858. (Eliseeva S., Sementsov D. // Phys. Sol. St. 2012. V. 54. N 10. P. 1981.)

[25] Eliseeva S.V., Ostatochnikov V.A., Sementsov D.I. // J. Magn. Magn. Mat. 2014. V. 354. P. 267.

[26] Abdi-Ghaleh R., Asad M. // Eur. Phys. J. D. 2015. V. 69. P. 13.

[27] Arce-Diego J.L., Lopez-Ruisanchez R., Lopez-Higuera J.M., Muriel M.A. // Opt. Lett. 1997. V. 22. N 9. P. 603.

[28] Геворгян А.А., Голик С.С. // ЖЭТФ. 2017. Т. 152. № 1. C. 30. (Gevorgyan A.H., Golik S.S. // J. Exp. Theor. Phys. 2017. V. 125. P. 22-34.

[29] Gevorgyan A.H., Golik S.S. // J. Mag. Mag. Mat. 2017. V. 439. N 1. P. 320.)

[30] Moocarme M., Proscia N.V., Vuong L.T. // Sci. Rep. 2017. V. 7. P. 40718. 